\title{
DIGITAL TWINS PARA SUSTENTABILIDADE E GESTÃO DE ACIDENTES
}

\author{
Ricardo Pontes Resende ${ }^{(1)}$, Bruno Mataloto ${ }^{(1)}$, Luís Dias ${ }^{(1)}$, João Carlos Ferreira ${ }^{(1)}$, \\ Vasco Rato $^{(1)}$, João Bone ${ }^{(2)}$
}

(1) ISTAR - Instituto Universitário de Lisboa

(2) Inov INESC Inovação, Lisboa

\begin{abstract}
Resumo
Os modelos BIM são maioritariamente gerados e usados no contexto do projeto e construção. Contudo, a informação que contêm é cada vez mais utilizada na gestão dos edifícios e cidade. Este trabalho descreve o desenvolvimento de ferramentas baseadas em modelos BIM que são enquadradas no conceito de Smart City. Estas aplicações contêm informação geométrica e funcional da construção e trocam dados com outras fontes tal como sensores ambientais, de consumo de energia ou água ou com os responsáveis técnicos e utilizadores, tornando-se em Gémeos Digitais/Digital Twins.

São apresentadas duas aplicações onde são usados modelos BIM de pequena e grande escala como suporte de visualização e interação com os utilizadores. Em ambas são feitas integrações de informação de várias origens que são integradas numa plataforma agregadora.

Nestas aplicações o modelo BIM é uma das peças do sistema que compreende sensores, bases de dados, algoritmos de filtragem e tratamento de dados, sendo por isso necessário recorrer a técnicas de extração, compatibilização de informação de várias fontes e apresentação ao utilizador em suportes acessíveis, o que aponta caminhos para a utilização do BIM em novos cenários.
\end{abstract}

\section{Introdução}

A utilização de modelos BIM fora do contexto do projeto e construção tem crescido significativamente. A gestão eficiente do património público construído foi, aliás, uma das motivações para a implementação do BIM no Reino Unido[1], que iniciou em 2018 o ambicioso programa "National Digital Twin Programme", uma parceria entre o governo britânico e a Universidade de Cambridge [2].

O que distingue um Digital Twin (DT) de um modelo BIM tradicional é a sua ligação a fontes de informação exteriores, com as quais troca dados. Assim, o DT informa sobre o estado atual da instalação, edifício ou outra infraestrutura, e permite análises, simulações de cenários ou a análise retrospetiva de eventos passados. Estas capacidades permitem a gestão e decisão 
informadas, com melhor economia de tempo e recursos e desempenho superior [3], [4]. Os cenários de uso são infindáveis. O DT das urgências de um hospital pode obter o número de utentes em espera do sistema de gestão clínica e extrair a capacidade da sala de espera do modelo BIM, tendo em conta a área e volume da sala, número de cadeiras e ainda a taxa de renovação de ar atual a partir dos equipamentos em funcionamento. Ao relacionar estes dados, o DT da urgência pode propor a abertura de um espaço alternativo para assegurar conforto ou distanciamento mínimo entre utentes, ou, em sentido inverso, encerrar parte do espaço, minimizando custos de funcionamento. $\mathrm{O}$ modelo 3D mostra visualizações de fácil compreensão ao enfermeiro responsável pelo turno, permitindo-lhe decisões informadas. Podem ser adicionados modelos preditivos baseados na expectativa de influxo de utentes, pessoal médico em serviço e até a meteorologia.

O DT pode ser visto como uma evolução em relação aos modelos BIM tradicionalmente usados em projeto e obra. Em muitas empreitadas os modelos são atualizados regularmente com os trabalhos realizados ou alterações feitas em obra para produzir o as built. Se o modelo é ligado a fontes de informação que o atualizam de forma autónoma e é usado para previsões ou simulações podemos considerar que estamos perante um DT.

A utilização de um DT tem de ser mais flexível que a de um modelo BIM tradicional. Em primeiro lugar, o DT contém características e funcionalidades que não são cobertas por aplicações de desenvolvimento de modelos, como o Archicad ou o Revit. Os registos de temperatura de um espaço, o histórico de manutenção de um equipamento ou a energia produzida por um painel solar numa cobertura são exemplos de dados que devem ser relacionados com os ativos que lhes deram origem (par sensor/local) mas não devem ficar guardados dentro do modelo BIM. Em segundo lugar, múltiplos utilizadores devem poder usar o modelo simultaneamente através de interfaces simplificados e em computador, tablet, smartphone ou em Realidade Aumentada/Virtual. A opção mais frequente é basear os DT na cloud, usando plataformas da indústria como a Autodesk Forge, ou construídas de raiz, como se verá adiante.

Outra característica importante dos DT é que as equipas de desenvolvimento envolvem profissionais de diferentes áreas: Eng. Informáticos e de Telecomunicações que desenvolvem e mantêm os sensores, redes de comunicação, bases de dados, plataformas cloud, integração e ligação entre sistemas e a segurança dos dados, especialistas em usabilidade e desenvolvimento de produto.

Enquanto o modelo BIM comum descreve um edifício, um DT pode descrever um complexo como um campus, um hospital, um bairro ou mesmo uma cidade. Assim, o DT pode ser um ecossistema de (sub) modelos que usam tecnologias diferentes e trocam dados de diferentes tipos. Este cenário ainda não é ainda comum, mas é um dos objetivos futuros (Figura 1). 


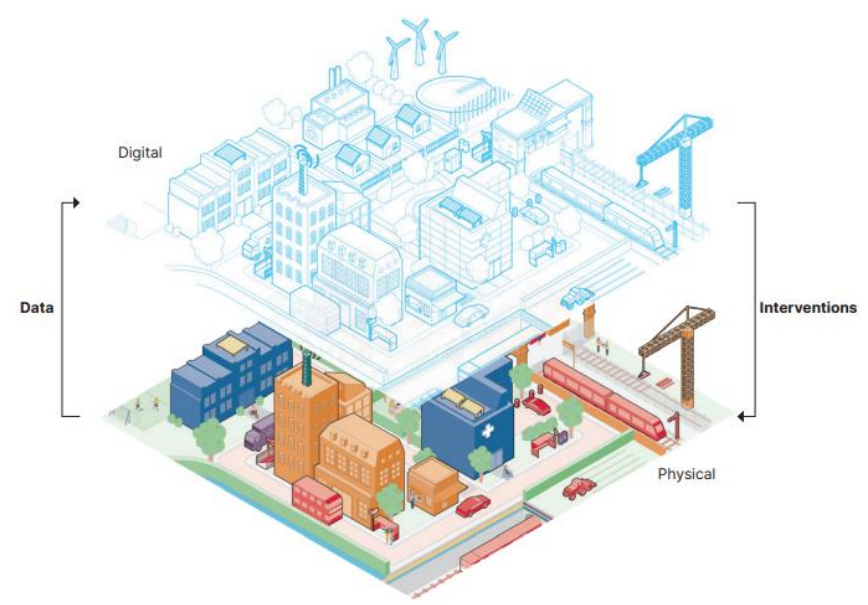

Figura 1: Interação entre DT e ativos[2].

Este trabalho descreve de forma sumária duas aplicações em desenvolvimento no centro de investigação ISTAR - Instituto Universitário de Lisboa. A primeira aplicação é um piloto que tem como objetivo caracterizar e melhorar as práticas ambientais dos utilizadores de edifícios públicos com o objetivo de reduzir o consumo de energia para iluminação e aquecimento/arrefecimento. Para tal foi instalada uma rede de sensores ambientais que são integrados no modelo BIM. A segunda aplicação visa aumentar a resiliência das cidades a desastres industriais e consiste num modelo urbano que inclui equipamentos sociais importantes e a instalação fabril. A aplicação integra-se numa plataforma com outras funcionalidades e facilita a comunicação com os cidadãos.

A estrutura deste trabalho é a seguinte: após uma apresentação dos conceitos essenciais, apresentam-se as duas aplicações em secções independentes, finalizando-se com uma análise crítica e perspetivas futuras de desenvolvimento.

\section{Plataforma para a sustentabilidade de campus universitário}

O projeto "University Community Engagement in Technologies for Sustainability", financiado pela Fundação Calouste Gulbenkian tem como objetivo "fomentar a alteração dos comportamentos dos utilizadores do campus do ISCTE-IUL para reduzir o consumo de energia e melhorar a sustentabilidade ambiental do campus. Conjuga conhecimentos da Psicologia, Engenharia, Arquitetura e Informática, envolvendo toda a comunidade ISCTE-IUL e serão implementadas estratégias de economia comportamental e gamification" [5], [6].

Este projeto começa com a recolha em tempo real de dados de sensores de temperatura, humidade, ruído, ocupação e energia elétrica (Figura 2) e a recolha e análise numa plataforma IoT (Figura 3), que não só caracteriza o estado atual dos espaços instrumentados, mas também identificar padrões e comportamentos. Após a recolha, armazenamento e tratamento os dados são apresentados tanto em dashboards tradicionais como em modelos BIM dos espaços, retirados dos modelos do campus desenvolvidos pela Unidade de Edifícios e Recursos (UER) que tem vindo a desenvolver modelos BIM para gestão do campus que (Figura 4) [7]. 


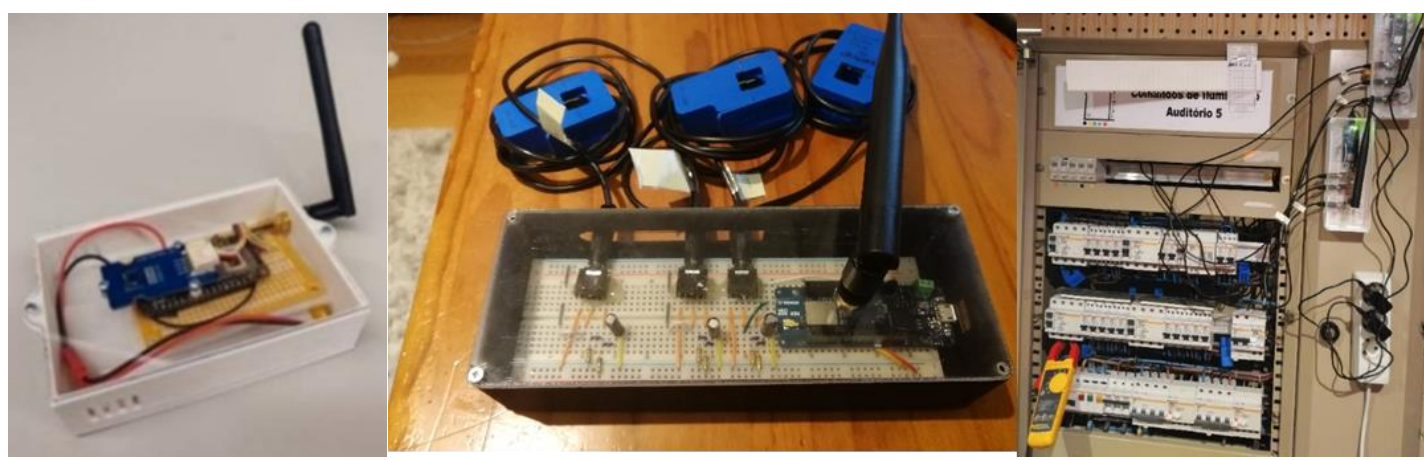

Figura 2: Sensores e placas de armazenamento e comunicação desenvolvidos: temperatura, humidade e ruído (esq.), de energia elétrica (centro) e instalação em quadro de auditório (dir.).

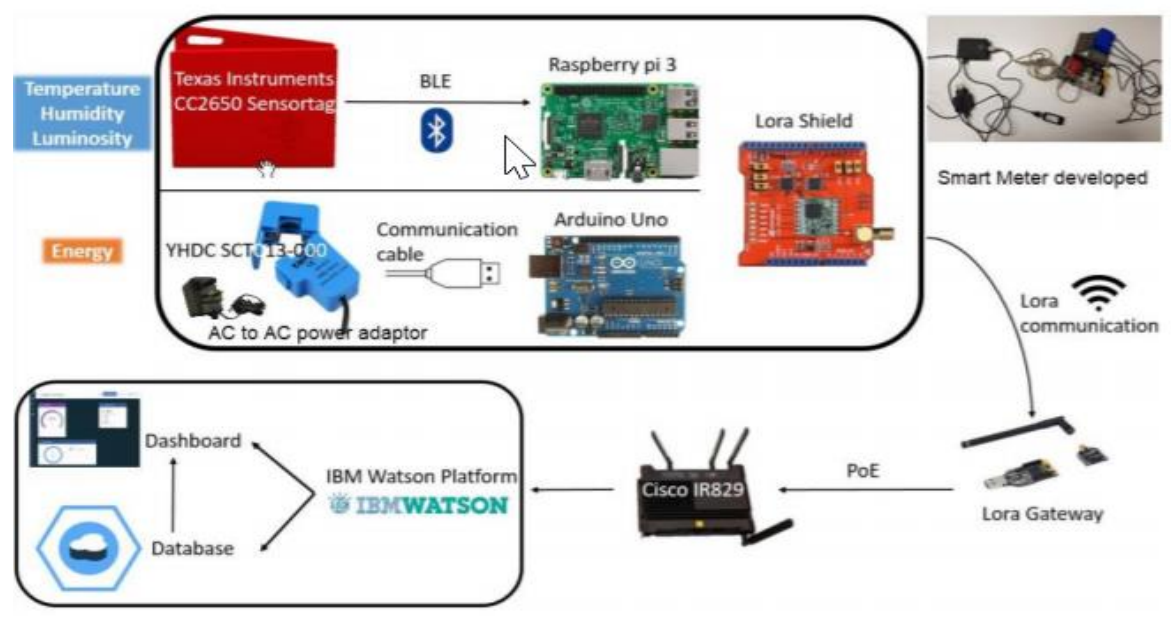

Figura 3: Tecnologia de recolha e telecomunicação de dados baseada em rede Lora.

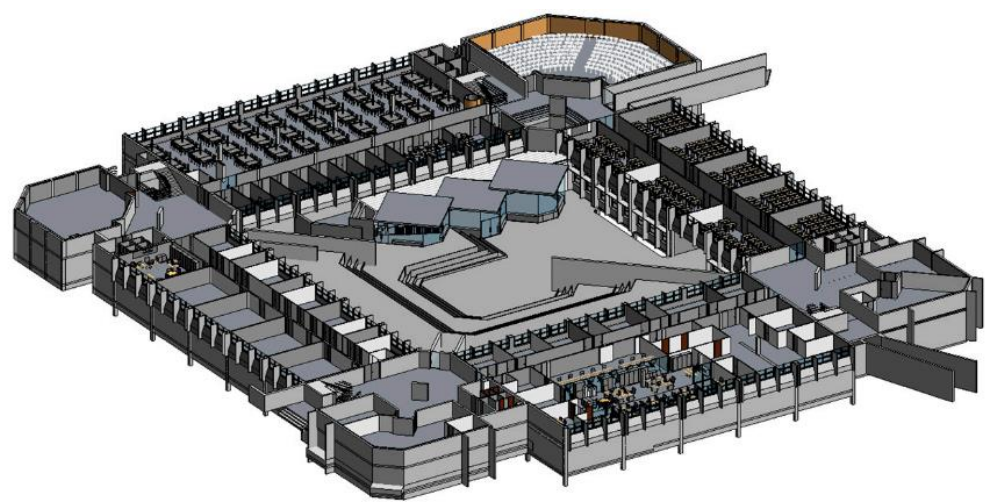

Figura 4: Vista do modelo do edifício 1 do ISCTE-IUL ao nível do piso 1.

As Figuras 5 a 8 apresentam os dois tipos de interface desenvolvidos. A Figura 5 apresenta o dashboard tradicional que representa a temperatura e humidade em seis locais de uma sala de servidores, incluindo valores limite e gráficos do histórico. As Figuras 6 a 8 apresentam a mesma informação para um espaço aberto de trabalho e atendimento ao público, num interface baseado em BIM. A figura 6 representa a intensidade de luz detetada por cada sensor, o que 
permite controlar se há iluminação artificial desnecessária. As Figuras 7 e 8 apresentam a humidade relativa e temperatura, respetivamente. As cores dos espaços correspondem à grandeza representada, sendo representados a encarnado os valores fora das condições de conforto definidas para o espaço ou outros avisos definidos no back end. Os sensores instalados no exterior são representados por esferas. A plataforma tem critérios de poupança embebidos: na Figura 8 há um alarme pois foi detetado que o ar condicionado, que neste espaço é controlado pelo utilizador, está ligado sem necessidade.

A interface baseada em BIM permite aos leigos a navegação no modelo e compreensão intuitiva e ainda a comparação dos valores correntes com o histórico diário, através da barra do tempo na base, ou diário, através do calendário. A ligação à base de dados é feita em tempo real e quando a navegação temporal não está a ser usada os dados são atualizados de acordo com a frequência de transmissão definida na rede de sensores.

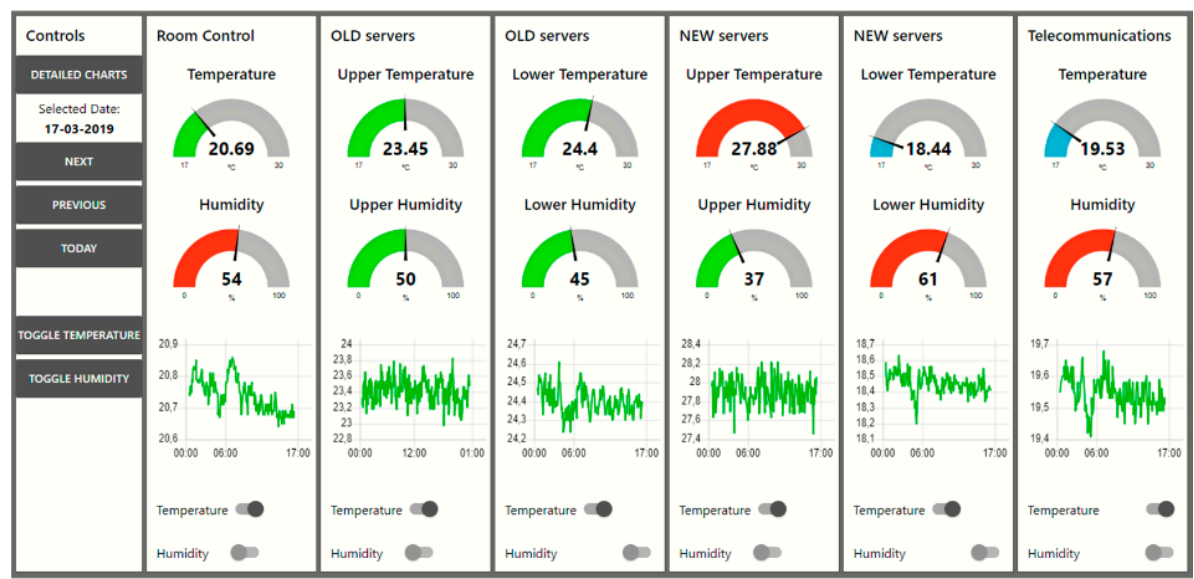

Figura 5: Dashboard de visualização de temperatura e humidade na sala de servidores [8].

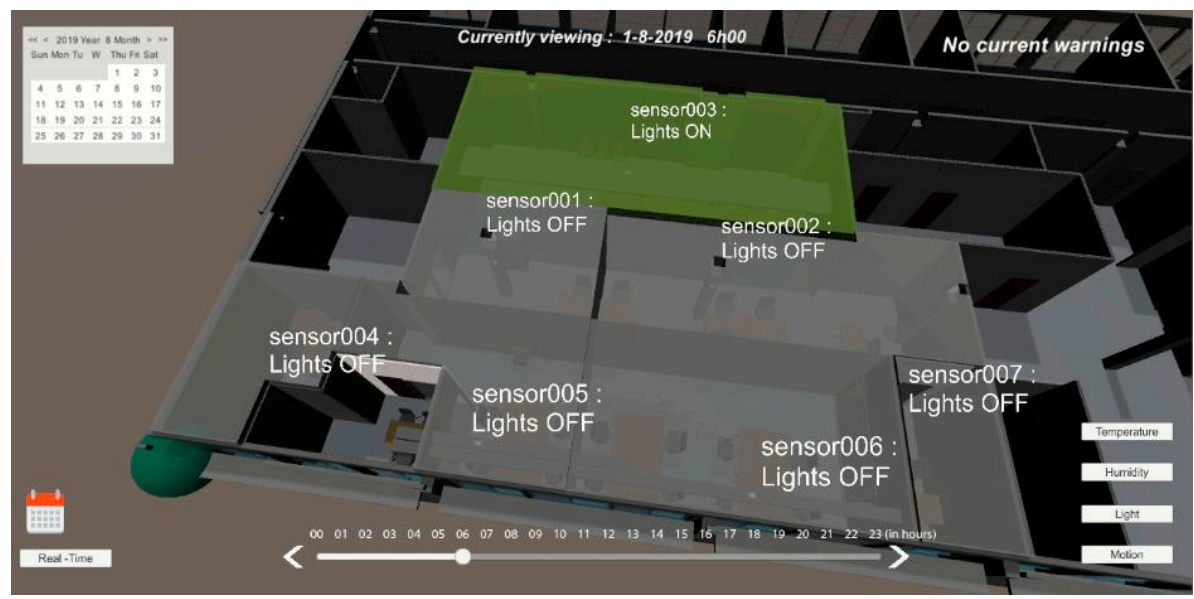

Figura 6: Interface BIM de visualização de intensidade de iluminação em espaço de trabalho e de atendimento ao público [8]. 


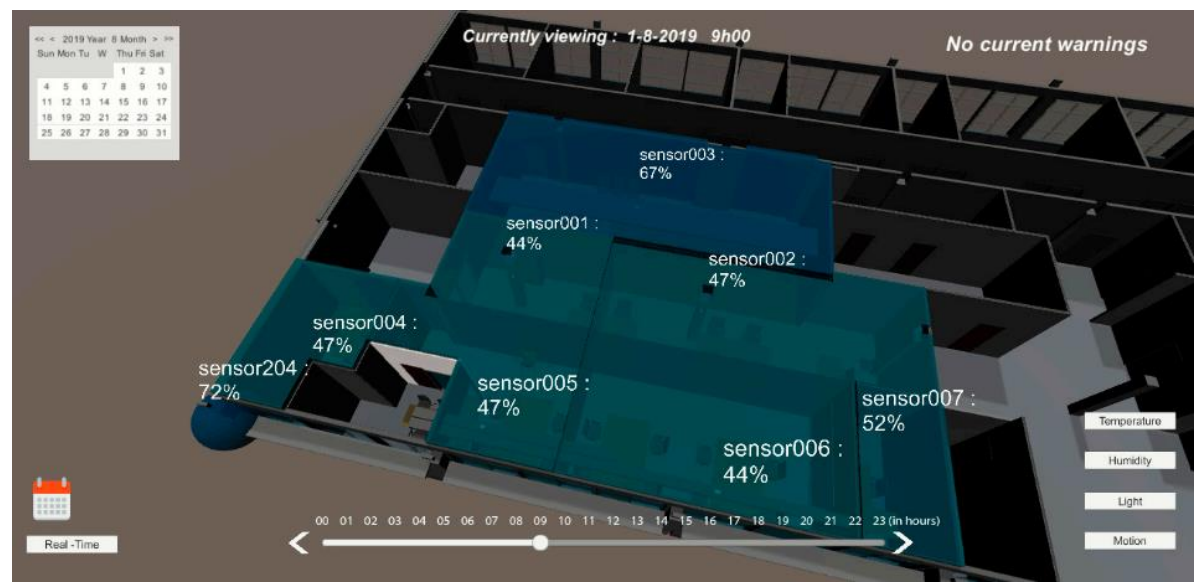

Figura 7: Interface BIM de visualização de humidade relativa em espaço de trabalho e de atendimento ao público [8].

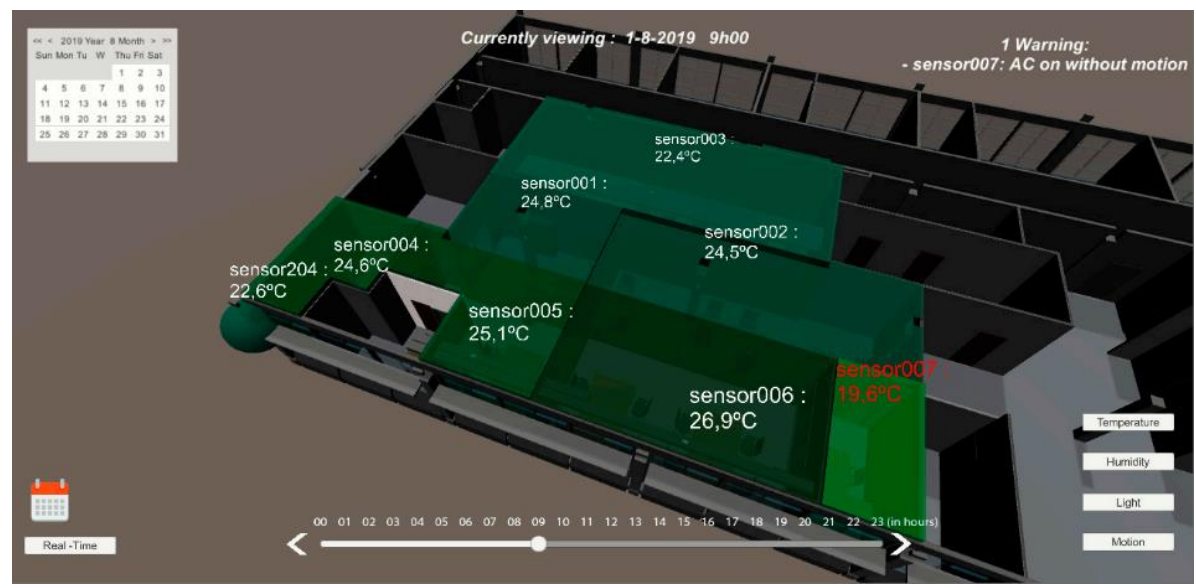

Figura 8: Interface BIM de visualização de temperatura do ar em espaço de trabalho e de atendimento ao público, com aviso no canto superior direito [8].

\subsection{Integração do modelo BIM}

Para a integração do modelo na plataforma, começou por se inserir os sensores no modelo BIM do edifício desenvolvido no Autodesk Revit, versão 2019. Para tal foram construídas famílias da categoria Specialty Equipment. A geometria da família reproduz de forma aproximada o sensor ambiental (Figura 9) e os parâmetros incluem as características do sensor (type parameters), e as identificações necessários à sua identificação na rede e base de dados (instance parameters). O modelo foi usado para escolher os locais de instalação, afastados das janelas, não afetados diretamente por equipamentos de renovação de ar ou de climatização e pela limpeza ou uso do espaço. Extraiu-se do modelo do edifício as salas a representar e, usando rotinas de Dynamo, foram geradas massas correspondentes à região afeta a cada sensor e ficheiros XML com a informação correspondente a cada sensor. Para os sensores exteriores a rotina gera massas com forma esférica.

O modelo é exportado para o motor de jogos Unity [9] onde é adicionado o calendário e restantes elementos gráficos e textuais do User Interface (UI) e foram escritas rotinas de C\# para identificar as massas correspondentes aos sensores na base de dados SQL. O Unity permite 
produzir aplicações que correm em praticamente todos os ambientes e plataformas: Windows, Android, iOS, web, etc. A aplicação foi compilada para Web, para poder ser acedida num browser pelos funcionários que trabalham no espaço, e Android, para correr num tablet a instalar num quiosque eletrónico na sala.

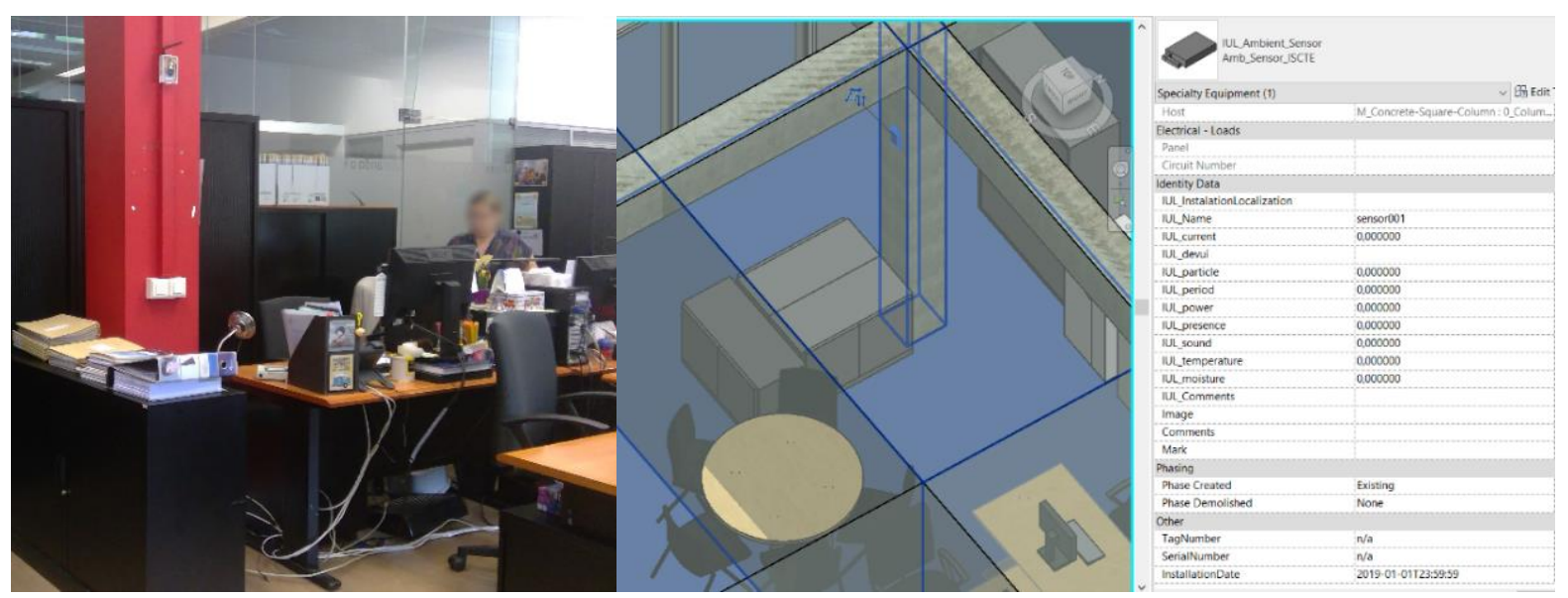

Figura 9: Sensor instalado no pilar (esq.) e modelação mostrando parâmetros do tipo Instance (dir.).

\subsection{Conclusões}

Esta aplicação insere-se na dimensão 6D do BIM, que "envolve a inclusão de informação para suportar a gestão das instalações para conduzir a melhores resultados operacionais" [10]. Os objetivos são três: a poupança energética imediata no campus, a mudança de hábitos dos utentes que se irá repercutir noutros ambientes, o desenvolvimento e teste de novas plataformas e a investigação da sua eficácia na mudança de hábitos. É importante referir que todas as questões relacionadas com a privacidade e segurança de dados foram acauteladas, tendo todo o trabalho sido validado pela Comissão de Ética do ISCTE-IUL.

Neste piloto a aplicação tem uma escala pequena, tendo sido instalados sensores em quatro espaços do ISCTE. Devido aos baixos custos dos sensores, que funcionam autonomamente durante cerca de dois anos e da rede Lora, a solução pode ser aplicada em maior escala. O nível de detalhe do modelo é adaptado aos objetivos. O detalhe visual (cores, texturas, mobiliário, equipamento) facilita a colocação dos sensores e dá aos utilizadores maior envolvimento. A informação contida na construção (paredes, pavimentos, iluminação) e equipamentos pode ser usada para simulações energéticas ou cálculos de ventilação ou outros que apoiam a interpretação dos resultados e a tomada de decisão.

\section{Aplicação para gestão de acidentes industriais}

As indústrias pesadas em centros urbanos preocupa as populações e decisores, não só devido à contaminação da atmosfera, solo e água mas também devido à possibilidade de acidentes industriais. O projeto "Infrastress - Improving resilience of sensitive industrial plants \& infrastructures exposed to cyber-physical threats, by means of an open testbed stress-testing system" [11], com um financiamento total de M€7,9 envolve 27 parceiros de 11 países europeus 
e desenvolve uma estrutura integrada que inclui a deteção e análise de ameaças físicas e cibernéticas, perceção situacional e promoção da resiliência. Um dos cinco pilotos envolve o Inov (https://www.inov.pt/), de quem o ISTAR é parceiro, a Câmara Municipal do Barreiro e a SGL Carbon (https://www.sglcarbon.com), proprietária da fábrica da Fisipe no Barreiro. Esta é uma instalação industrial com a classificação Seveso [12], ou seja, utiliza ou armazena produtos químicos ou petroquímicos perigosos.

Decidiu-se integrar na plataforma de gestão de acidentes uma representação visual e funcional da fábrica e da sua envolvente urbana. Esta representação, elaborada na plataforma Unity, está na fronteira entre um modelo BIM e SIG (Sistema de Informação Geográfica), pois a escala abarca não um edifício, mas um complexo com vários edifícios que são descritos geometricamente de forma simplificada. A aplicação BIM é uma parte da aplicação, que integra outras valências de gestão de crises (Figura 9). A comunicação da aplicação BIM/Unity com a plataforma é feita através do protocolo REST.

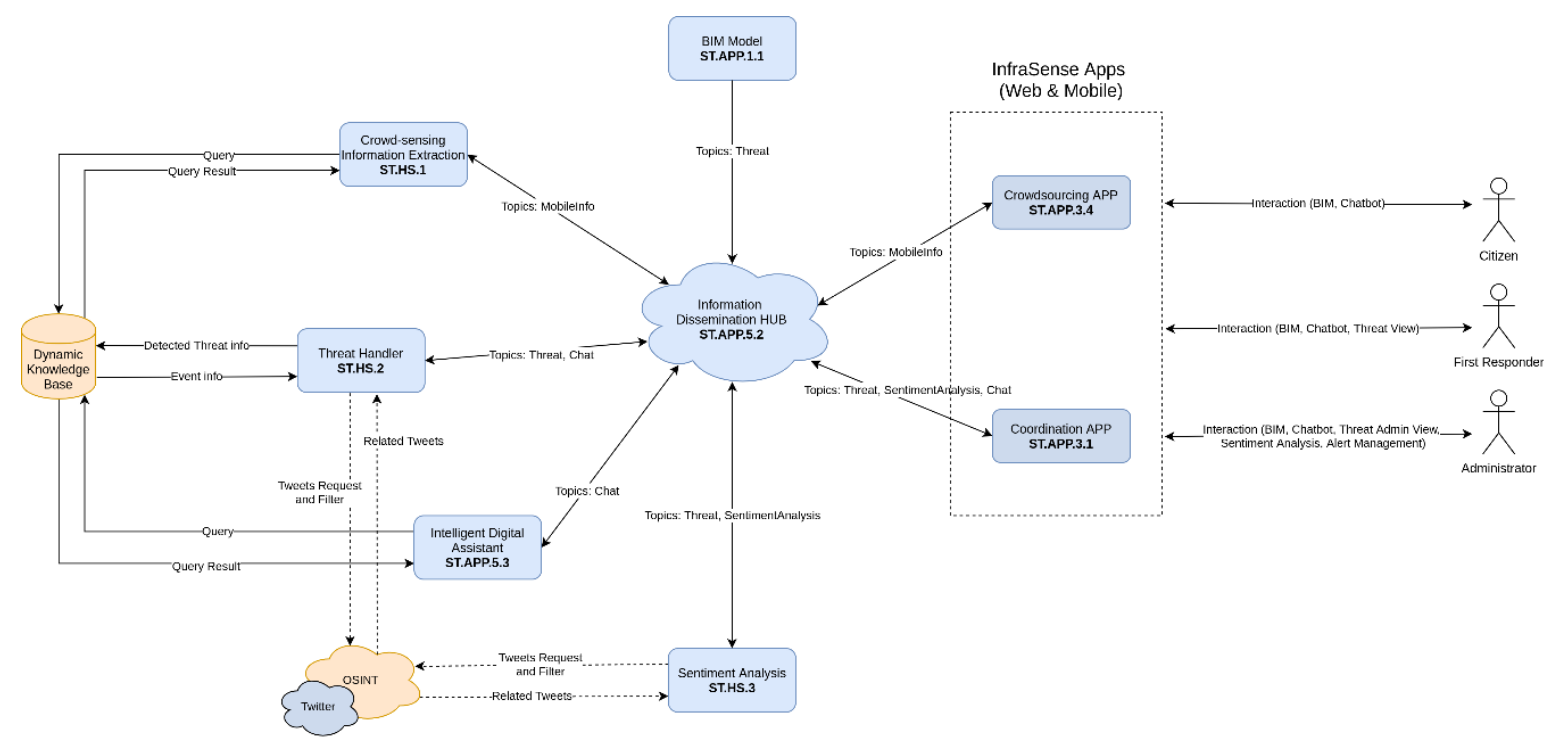

Figura 9: Arquitectura de desenvolvimento do Digital Twin das instalações da Fisipe.

Do ponto de vista geométrico, a aplicação apresenta uma vista aérea do território, com cerca de 30 ha, e massas correspondentes à volumetria dos edifícios fabris, que são enriquecidas com os parâmetros relevantes para cenários de acidente (Figura 10). Algumas das características são, para além da identificação e coordenadas globais e locais, o tipo de riscos (explosão, incêndio, fuga de substância perigosa), a reação perante acidentes próximos (neutro, auxílio ou agravante) e acidentes ativos.

A aplicação, que corre em desktop e smartphone, permite a um cidadão inserir acidentes nos edifícios ou em qualquer ponto do território, e também votar positiva ou negativamente nos acidentes, à semelhança da aplicação Waze. Quando um acidente é selecionado, os equipamentos num raio de $50 \mathrm{~m}$ são analisados e mudam de cor consoante sejam neutros, agravantes ou auxiliares a esse tipo de acidente. Por exemplo, se houver um fogo, um equipamento de extinção na vizinhança passará a verde e um material inflamável a encarnado. Todas as ações dos utilizadores são apreciadas pelo administrador, membro da Proteção Civil, com controlo total sobre a plataforma e conhecimento das informações de outras fontes. 


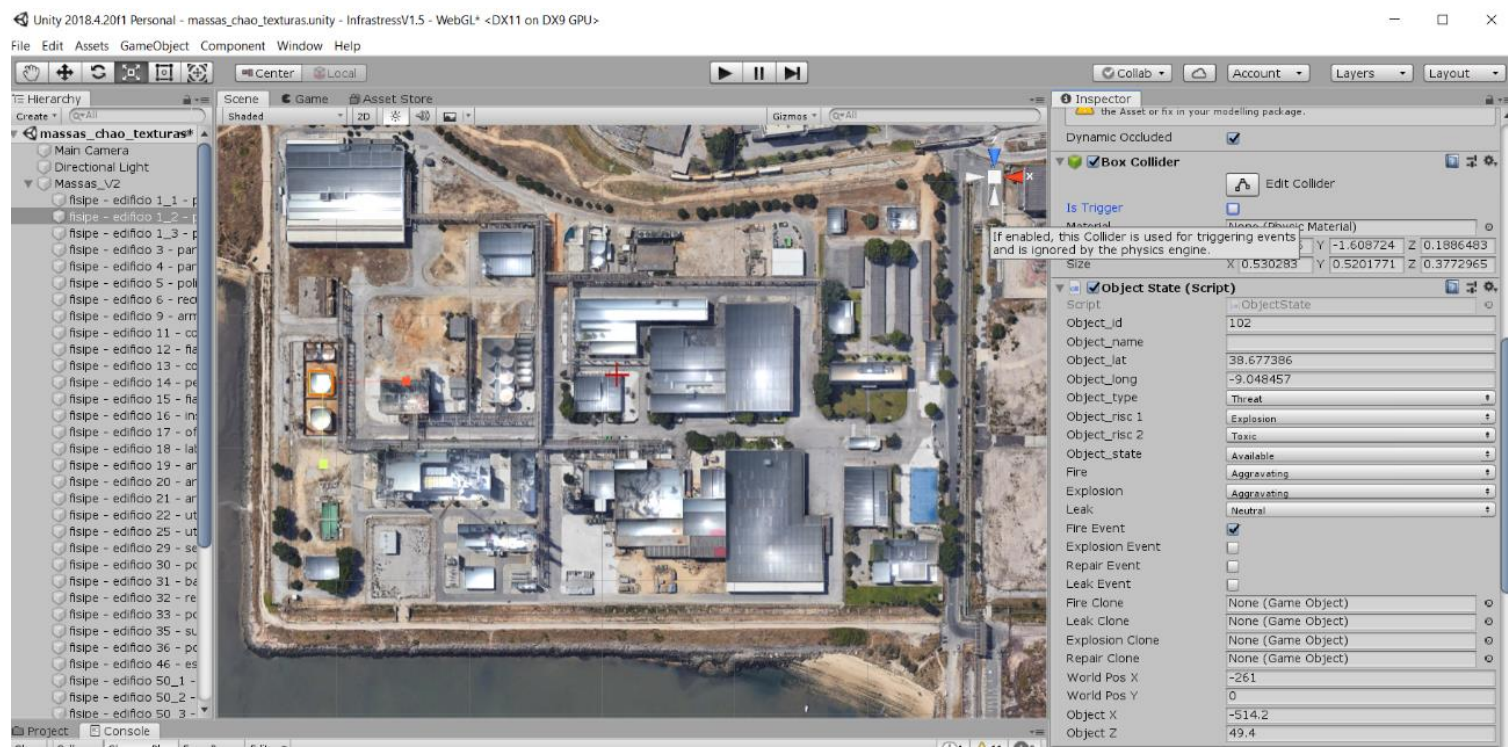

Figura 10: Ambiente de desenvolvimento do Digital Twin da Fisipe em Unity.
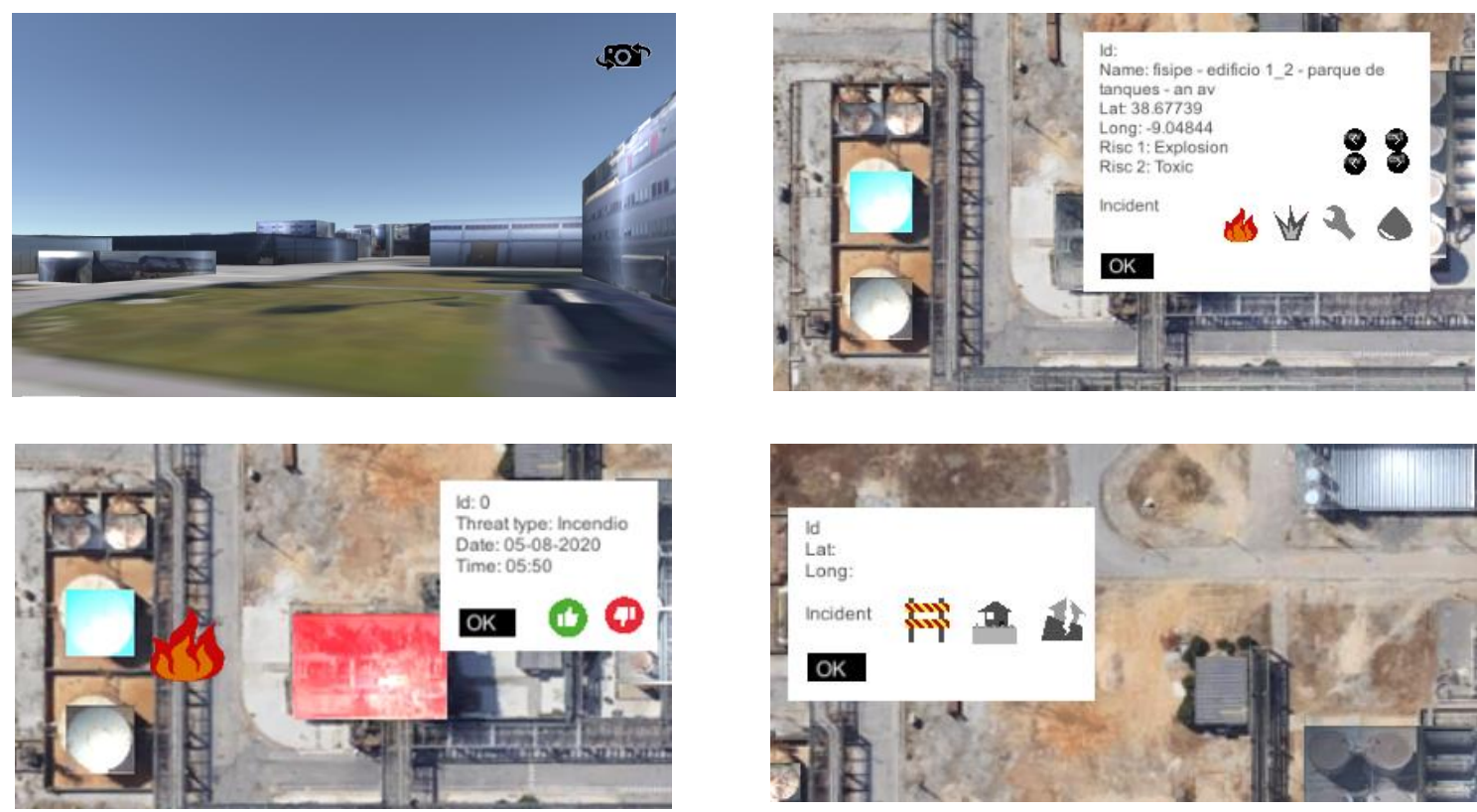

Figura 11: Vista de chão do modelo (topo esq.), inserção de acidente num tanque (topo dir.), votação de reforço em acidente e equipamento em perigo (base esq.), adição de barreira em estrada (base dir.).

\subsection{Conclusões}

Esta aplicação tem várias particularidades. Pela sua escala não pode ser considerada BIM "puro", apesar de aliar uma representação geométrica do mundo real com dados que são manipulados e usados para um objetivo. Por outro lado, insere-se numa plataforma sofisticada que agrega informação de inúmeras fontes, como as redes sociais e os canais de emergência. Finalmente, aplica-se a um uso pouco usual no BIM, a gestão de catástrofes. Este trabalho 
multidisciplinar responde ao interesse de outras áreas pelo BIM, havendo interesse em reproduzir a metodologia no interior de edifícios.

\section{Conclusões}

É já conhecimento comum que os modelos digitais dos empreendimentos têm utilidade para além da fase inicial da vida. Este trabalho apresenta dois exemplos de diferentes escalas e objetivos, que servem o mesmo propósito: suportar a fase de utilização dos equipamentos. Em ambos os casos a representação geométrica é usada para facilitar a interação dos utentes com a aplicação, embora no primeiro caso o objetivo seja a melhoria da sustentabilidade através da baixa do consumo energético e no segundo caso a gestão de acidentes industriais.

Realça-se a crescente facilidade de integração dos modelos BIM em plataformas como o Unity, que tem como uma das suas quatro áreas a Construção (sendo as restantes os Jogos, Animação e Automóvel), que permitem a importação de modelos BIM, a fácil distribuição da aplicação e não facilitam aspeto gráfico de boa qualidade sem recurso a programação. Persiste, no entanto, a necessidade ter conhecimentos de programação para trocar informação com aplicações externas, e para implementar as funcionalidades de interação com o modelo.

\section{Agradecimentos}

Os autores agradecem o financiamento da Fundação Calouste Gulbenkian através do Programa Sustentabilidade e à Unidade de Edifícios e Recursos pela autorização para a instalação de sensores e utilização do modelo do Edifício Sedas Nunes do campus do ISCTE-IUL.

Os autores agradecem o apoio financeiro do Programa Horizonte 2020 da União Europeia ao projeto Infrastress através do contracto de financiamento n ${ }^{\circ} 833088$. Os autores estão também reconhecidos aos parceiros nacionais do projeto Infrastress Inov, SGL Carbon e Câmara Municipal do Barreiro.

Este trabalho é parcialmente financiado por fundos nacionais através da FCT - Fundação para a Ciência e Tecnologia, I.P., projeto FCT UIDB/04466/2020.

\section{Referências}

[1] "Construction 2025: strategy - $\quad$ GOV.UK." https://www.gov.uk/government/publications/construction-2025-strategy (accessed Aug. 06, 2020).

[2] "National Digital Twin Programme | Centre for Digital Built Britain." https://www.cdbb.cam.ac.uk/what-we-do/national-digital-twin-programme (accessed Aug. 03, 2020).

[3] R. Woodhead, P. Stephenson, and D. Morrey, "Digital construction: From point solutions to IoT ecosystem," Automation in Construction, vol. 93, pp. 35-46, Sep. 2018, doi: 10.1016/j.autcon.2018.05.004.

[4] M. Grieves, "Origins of the Digital Twin Concept," vol. 23, no. August, pp. 889-896, doi: 10.13140/RG.2.2.26367.61609. 
[5] "Technology for Sustainability | Fundação Calouste Gulbenkian." https://gulbenkian.pt/project/technology-for-sustainability/ (accessed Aug. 04, 2020).

[6] B. M. G. Mataloto, "Iot*(Ambisense) - Smart Environment Monitoring Using Lora," 2019.

[7] R. Resende et al., "Plataforma Web-BIM para Gestão de Instalações de um Campus Universitário," in $1^{\circ}$ Congresso Português de Building Information Modelling, 2016, pp. 501-511, [Online]. Available: http://ptbim.org/.

[8] B. Mataloto, J. C. Ferreira, R. Resende, R. Moura, and S. Luís, "BIM in People2People and Things2People Interactive Process," Sensors, vol. 20, no. 10, p. 2982, May 2020, doi: $10.3390 / \mathrm{s} 20102982$.

[9] “Unity Real-Time Development Platform | 3D, 2D VR \& AR Engine.” https://unity.com/ (accessed Aug. 05, 2020).

[10] "BIM dimensions - 3D, 4D, 5D, 6D BIM explained | NBS." https://www.thenbs.com/knowledge/bim-dimensions-3d-4d-5d-6d-bim-explained (accessed Aug. 05, 2020).

[11] “Critical Infrastructure | Infrastress." https://www.infrastress.eu/ (accessed Aug. 05, 2020).

[12] "EUR-Lex - 121215 - EN - EUR-Lex." https://eur-lex.europa.eu/legalcontent/PT/TXT/?uri=LEGISSUM\%3A121215 (accessed Aug. 05, 2020). 